\title{
Impact of Slow Blood Filling via Collaterals on Infarct Growth: Comparison of Mismatch and Collateral Status
}

\author{
Jeong Pyo Son, ${ }^{\mathrm{a}, \mathrm{b}} \mathrm{Mi}$ Ji Lee, ${ }^{\mathrm{c}}$ Suk Jae Kim, ${ }^{\mathrm{c}}$ Jong-Won Chung, ${ }^{\mathrm{c}}$ Jihoon Cha, ${ }^{\mathrm{d}}$ Gyeong-Moon Kim, \\ Chin-Sang Chung, ${ }^{\mathrm{c}}$ Kwang Ho Lee, ${ }^{\mathrm{c}}$ Oh Young Bang, ${ }^{\mathrm{a}, \mathrm{c}}$ \\ aDepartment of Health Sciences and Technology, Samsung Advanced Institute for Health Sciences and Technology (SAIHST), Sungkyunkwan University, Seoul, \\ Korea \\ ${ }^{b}$ Center for Neuroscience Imaging Research (CNIR), Institute for Basic Science (IBS), Suwon, Korea \\ Departments of 'Neurology and 'Radioology, Samsung Medical Center, Sungkyunkwan University School of Medicine, Seoul, Korea
}

Background and Purpose Perfusion-diffusion mismatch has been evaluated to determine whether the presence of a target mismatch helps to identify patients who respond favorably to recanalization therapies. We compared the impact on infarct growth of collateral status and the presence of a penumbra, using magnetic resonance perfusion (MRP) techniques.

Methods Consecutive patients who were candidates for recanalization therapy and underwent serial diffusion-weighted imaging (DWI) and MRP were enrolled. A collateral flow map derived from MRP source data was generated by automatic post-processing. The impact of a target mismatch (Tmax $>6 \mathrm{~s}$ /apparent diffusion coefficient (ADC) volume $\geq 1.8$, ADC volume $<70 \mathrm{~mL}$; and Tmax $>10 \mathrm{~s}$ for $\mathrm{ADC}$ volume $<100 \mathrm{~mL}$ ) on infarct growth was compared with MR-based collateral grading on day $7 \mathrm{DWl}$, using multivariate linear regression analysis.

Results Among 73 patients, 55 (75\%) showed a target mismatch, whereas collaterals were poor in $14(19.2 \%)$, intermediate in $36(49.3 \%)$, and good in $23(31.5 \%)$ patients. After adjusting for initial severity of stroke, early recanalization $(P<0.001)$ and the MR-based collateral grading $(P=0.001)$, but not the presence of a target mismatch, were independently associated with infarct growth. Even in patients with a target mismatch and successful recanalization, the degree of infarct growth depended on the collateral status. Perfusion status at later Tmax time points (beyond the arterial phase) was more closely correlated with collateral status.

Conclusions Patients with good collaterals show a favorable outcome in terms of infarct growth, regardless of the presence of a target mismatch pattern. The presence of slow blood filling predicts collateral status and infarct growth.

Keywords Stroke; Collateral circulation; Magnetic resonance imaging
Correspondence: Oh Young Bang Department of Neurology, Samsung Medical Center, Sungkyunkwan University, 81 Irwon-ro, Gangnam-gu, Seoul 06351, Korea

Tel: +82-2-3410-3599

Fax: +82-2-3410-0052

E-mail: neuroboy50@naver.com

Received: July 27, 2016

Revised: August 30, 2016

Accepted: September 22, 2016

This research was supported by a grant of the Korea Health Technology REtD Project through the Korea Health Industry Development Institute (KHIDI), funded by the Ministry of Health \&t Welfare, Republic of Korea (HI14C1531 and HC15C1056).

The authors have no financial conflicts of interest.

\section{Introduction}

The use of magnetic resonance imaging (MRI) to predict the response to early recanalization therapy and to identify patients for delayed treatment is attractive. However, recent trials have showed controversial results that selecting patients using a mismatch paradigm is a useful technique for identifying patients who would differentially benefit from recanalization therapy. ${ }^{1-3}$ Continuous efforts have been made by researchers to determine the optimal definition of a target mismatch pattern, such as the mismatch ratio and Tmax threshold. ${ }^{4-9}$

In patients with acute ischemic stroke, both antegrade flow and (delayed) retrograde collateral flow maintain cerebral perfusion within ischemic regions. We have reported that the angio- 
graphic collateral grade determines the rate of recanalization, hemorrhagic transformation, and infarct growth after revascularization therapy. ${ }^{10-12}$ Patients with poor collateral flow have shown a low recanalization rate regardless of the mode of revascularization therapy or the site of the occlusion. ${ }^{11}$ Even if recanalization is achieved after revascularization therapy, these patients often experience clinical deterioration due to symptomatic hemorrhagic transformation. ${ }^{12}$ Recanalization is only related to a positive clinical outcome if adequate collateralization prevents infarction until the vessel is recanalized, ${ }^{10,13}$ and a good collateral status could feasibly extend the time window for endovascular procedures. ${ }^{14,15}$

Our hypothesis is that delayed perfusion (slower blood filling at later Tmax time points) via collaterals better predicts infarct growth than the perfusion status at earlier Tmax time points. In the present study, we evaluated the correlation between perfusion-diffusion mismatch and the collateral status. This was measured by a specific technique we developed to evaluate collateral flow using dedicated MRI sequences generated from magnetic resonance perfusion (MRP) source data, which yields an excellent correlation with the collateral status graded using conventional angiography. ${ }^{16}$ In addition, the impact of slow blood filling via collaterals on infarct growth was compared with that of a target mismatch on MRP.

\section{Methods}

\section{Patient selection}

Using data from a prospectively maintained registry, we identified patients who were considered eligible for recanalization therapy for acute infarction within the middle cerebral artery (MCA) territory. Data from consecutive patients who were treated from June 2005 to December 2012 at a university medical center were analyzed retrospectively. Inclusion criteria for this study were: (1) subjects who presented within 6 hours of symptom onset; (2) subjects who underwent serial brain MRI, including diffusion-weighted imaging (DWI), MRP, and MR angiography (MRA), at admission and at day 7; (3) subjects who had a NIH Stroke Scale (NIHSS) score of 4 or more points at admission; and (4) subjects with internal carotid artery and/or proximal MCA (M1 segment) occlusion associated with symptoms on admission MRA (Supplementary Figure 1). This study was approved by the local institutional review board. All patients gave written informed consent for participation in the study.

Patients were evaluated based on demographic data, medical history, vascular risk factors, routine blood tests, brain imaging, and cardiological assessments, according to a protocol. Stroke mechanisms were subtyped using the trial of ORG 10172 in acute stroke treatment (TOAST) classification, ${ }^{17}$ and were diagnosed by the consensus of two stroke neurologists. Serial assessments of stroke severity using the NIHSS were performed during hospitalization and whenever neurological deterioration occurred.

\section{MR perfusion methods and image analysis}

MRI was performed using a 3T Philips Achieva MR scanner (Philips Medical Systems, Best, the Netherlands). Pretreatment DWI and MRP assessments were performed on all patients. Posttreatment MRP was also performed on all patients 7 days after treatment. Additional MRI scans were performed after any signs of clinical deterioration.

Typical MRI sequences for acute stroke included at least DWI, dynamic susceptibility contrast-enhanced MRP (DSC-MRP), fluid-attenuated inversion recovery, and MRA of the cervical and intracranial vessels (3-dimensional time-of-flight MRA and contrast-enhanced MRA, including extracranial carotid and vertebral arteries). DWI was obtained with two levels of diffusion sensitization (b values of 0 and $1,000 \mathrm{~s} / \mathrm{mm}^{2} ; 5-$ to $7-\mathrm{mm}$ slice thickness; and no gap). DSC-MRP was performed using gradientecho and echo-planar imaging techniques after administration of intravenous gadolinium (Dotarem (gadoterate meglumine); Guerbet, Aulnay-sous-Bois, France), with a repetition time of 1,718 milliseconds for a total acquisition time of approximately 90 seconds with 20 slices. Contrast agent was injected at a dose of $0.1 \mathrm{mmol} / \mathrm{kg}$ body weight with a flow rate of $3 \mathrm{~mL} / \mathrm{s}$ by a power injector into an antecubital vein via an 18-gauge intravenous cannula about 7 seconds after beginning the acquisition. Other parameters for DSC-MRP were as follows: echo time $=35$ $\mathrm{ms}$, flip angle $=40^{\circ}$, acquisition matrix $=128 \times 128$, field of view $=24 \times 24 \mathrm{~cm}^{2}$, section thickness $=5 \mathrm{~mm}$, and intersection gap $=2 \mathrm{~mm}$. In total, 1,000 DSC-MRP raw images, composed of 50 time points per slice, were obtained.

Slow blood filling was defined based on the perfusion parameter Tmax. Tmax is the time to the peak of the residue function map generated by deconvolution of the tissue concentration over the time curve, using an arterial input function from the contralateral MCA. ${ }^{18}$ MRP post-processing and data analysis were performed as described in our previous studies. ${ }^{19}$ MRI volume measurements were performed semiautomatically using a computerassisted volumetric analysis program (Medical Image Processing, Analysis and Visualization, Version 3.0; NIH, Bethesda, Md) by an investigator who was blinded to the clinical information.

The presence of a "target mismatch" pattern was defined based on the following criteria proposed in the Diffusion and Perfusion Imaging Evaluation For Understanding Stroke Evolution study-2 (DEFUSE-2) trial: (a) a ratio of 1.8 or more between the volumes of critically hypoperfused tissue ( $T \max >6 \mathrm{~s}$ ) and the ischemic 
core based on an apparent diffusion coefficient (ADC) threshold of $<600 \times 10^{-6} \mathrm{~mm}^{2} / \mathrm{s}$, with an absolute difference of $15 \mathrm{~mL}$ or more, (b) ischemic core volume of less than $70 \mathrm{~mL}$, and (c) less than $100 \mathrm{~mL}$ of tissue with a severe delay in bolus arrival $(\operatorname{Tmax}>10 \mathrm{~s})$.?

\section{Post-processing techniques to generate an MRP- derived collateral flow map}

Collateral flow maps were generated based on source data derived from DSC-MRP, as previously described. ${ }^{16}$ Collateral flow maps were automatically generated using a in-house software named FAST-COLL (Fast Analysis SysTem for COLLaterals) that was developed using MATLAB (MathWorks, Natick, MA). All these steps were typically completed within five minutes (Figure 1).

Criteria for the collateral flow map-based collateral grade were chosen based on the concept of the American Society of Interventional and Therapeutic Neuroradiology/Society of Interventional Radiology (ASITN/SIR) scale. Specifically, a poor grade (grade 1) was defined as no collaterals visible or slow collaterals (visible only in the late phase) to the part of the occluded MCA territory with persistence of some of the defect; intermediate grade (grade 2) was defined as rapid collaterals (visible in the mid to late phase) to the part of the occluded MCA territory with persistence of some of the defect; good grade (grade 3) was defined as slow but complete collateral flow to the occluded MCA territory; and excellent grade (grade 4) was defined as complete and rapid collateral flow to the vascular bed in the occluded MCA territory. Six axial slices of the collateral flow maps were used to assess leptomeningeal collateral grade. For statistical analysis, patients were divided into three groups, according to the grade: grade 1 (poor), grade 2-3 (intermediate), and grade 4 (good). Collateral flow images were reviewed independently by two investigators with knowledge of the symptomatic side and the site of occlusion. When the judgment of the two raters was inconsistent, a decision was made by consensus.

\section{Recanalization therapy and thrombolysis in cerebral infarction ( $\mathrm{TICl}$ ) grading}

The majority of the patients underwent endovascular treatment, including intra-arterial thrombolysis, angioplasty, stenting, and/or mechanical embolectomy, at the discretion of the attending physicians. Vascular reperfusion was based on the $\mathrm{TICl}$ classification, with assignments of 0 , no perfusion; 1 , penetration with minimal perfusion; $2 a,<67 \%$ perfusion; $2 b, \geq 67 \%$ perfusion; and 3 , complete perfusion of the affected vascular territory. ${ }^{20}$ In 13 patients who did not undergo endovascular treatment, $\mathrm{TICl}$ was determined with MRA at day 1. Twelve patients showed complete occlusion on follow up MRA and classified as having $\mathrm{TICl} 0 .{ }^{20}$ In one patient with partial spontaneous recanalization at day $1, \mathrm{TICl}$ grade could not be determined because timing of recanalization was not clear. $\mathrm{TICl}$ score was determined based on a consensus between two readers.

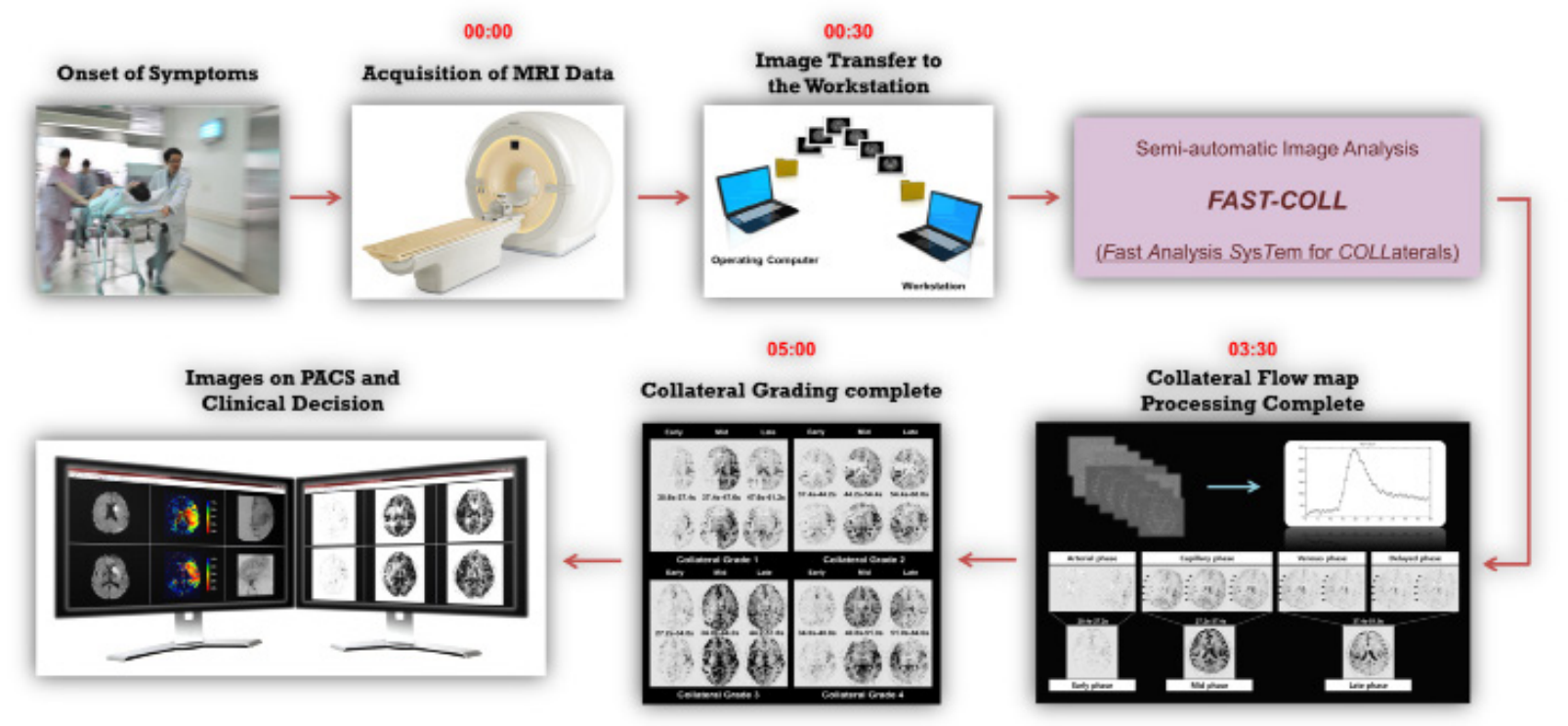

Figure 1. Basic scheme showing the FAST-COLL (Fast Analysis SysTem for COLLaterals) workflow. After the onset of symptoms, patients arrived at the hospital and the MRI data were acquired using typical MR sequences such as diffusion-weighted imaging (DWI), dynamic susceptibility contrast-enhanced magnetic resonance perfusion (DSC-MRP), and MR angiography (MRA). The MRI technician then transferred the MR raw data from the operating computer to the workstation using the file transfer program. The collateral flow map was automatically generated by FAST-COLL, and the stroke neurologist or radiologist evaluated the grade of collateral flow. All steps were typically completed within 5 minutes. The collateral flow map can also be easily viewed on the hospital picture archiving and communications system with other images. 


\section{Outcome measurements}

Infarct growth was defined as the difference between pretreatment lesion on ADC and final DWI infarct volume at day 7.

\section{Statistical analysis}

All data are presented as medians (25-75th percentile) or numbers (percentages), unless otherwise specified. The MannWhitney $\mathrm{U}$ test or Kruskal-Wallis test was used to compare continuous variables, and Pearson's chi-square test or Fisher's exact test was used to compare categorical variables between groups. We used the Bonferroni method to correct for multivariable comparisons. Predictors for infarct growth were first evaluated by univariate linear regression. Possible predictors that could influence infarct growth were age, baseline severity of stroke (NIHSS score and DWI infarct volume), recanalization (TICl scale), glucose level, time from MRI to recanalization therapy, the presence of the target mismatch pattern, and MRP-based collateral grade on pretreatment MRI. Multivariable linear regression mod-

Table 1. Patient characteristics depending on the MR perfusion-based collateral grading

\begin{tabular}{|c|c|c|c|c|}
\hline & \multicolumn{3}{|c|}{ Collateral grade } & \multirow{2}{*}{$P$ value } \\
\hline & Poor $(n=14)$ & Intermediate $(n=36)$ & Good $(n=23)$ & \\
\hline Female gender, n (\%) & $6(42.9)$ & $14(38.9)$ & $6(26.1)$ & 0.554 \\
\hline Age, year; mean (SD) & $69(59.8-73.5)$ & $65.5(53-73.8)$ & $65(48-72)$ & 0.581 \\
\hline \multicolumn{5}{|l|}{ Risk factors, n (\%) } \\
\hline Atrial fibrillation & $8(57.1)$ & $17(47.2)$ & 7 (30.4) & 0.240 \\
\hline Hypertension & $7(50.0)$ & $18(50.0)$ & $12(52.2)$ & 0.985 \\
\hline Diabetes & $5(35.7)$ & 7 (19.4) & $5(21.7)$ & 0.500 \\
\hline Hyperlipidemia & $2(14.3)$ & $11(30.6)$ & $6(26.1)$ & 0.568 \\
\hline Coronary artery disease & $1(7.1)$ & $4(11.1)$ & $4(17.4)$ & 0.717 \\
\hline Current smoking & - & $3(8.3)$ & 7 (30.4) & $0.017^{*}$ \\
\hline Prior stroke & $4(28.6)$ & $10(27.8)$ & $6(26.1)$ & 1.000 \\
\hline Stroke mechanism & & & & 0.054 \\
\hline Atherosclerotic & $1(7.1)$ & $10(27.8)$ & $13(56.5)$ & \\
\hline Cardioembolic & $9(64.3)$ & $17(47.2)$ & $8(34.8)$ & \\
\hline Other & $1(7.1)$ & $4(11.1)$ & $1(4.3)$ & \\
\hline Undetermined & $3(31.4)$ & $5(13.9)$ & $1(4.3)$ & \\
\hline Occlusion site, $\mathrm{n}(\%)$ & & & & 0.286 \\
\hline M1 & $11(78.6)$ & $20(55.6)$ & $12(52.2)$ & \\
\hline Distal internal carotid artery & $3(21.4)$ & $10(27.8)$ & $5(21.7)$ & \\
\hline Distal internal carotid artery+M1 & - & $6(16.7)$ & $6(26.1)$ & \\
\hline Initial NIHSS score, median (interquartile range [IOR]) & $18(15.8-21)$ & $13(9-16.8)$ & $12(10-15)$ & $0.002^{+}$ \\
\hline \multicolumn{5}{|l|}{ Pretreatment ischemic zone $(\mathrm{mL})$, median $(\mathrm{IQR})$} \\
\hline Initial diffusion-weighted imaging (DWI) lesion volume & $66.4(28.5-93.7)$ & $7.7(5.0-38.4)$ & $6.7(3.9-11.0)$ & $<0.001^{\dagger}$ \\
\hline $\operatorname{Tmax}>6 \mathrm{~s}$ & $174.5(149.3-240.8)$ & $119.4(58.7-155.0)$ & $40.7(24.2-78.0)$ & $<0.001^{\ddagger}$ \\
\hline $\operatorname{Tmax}>10 \mathrm{~s}$ & $121.7(92.9-180.9)$ & $52.8(25.5-85.7)$ & $9.2(2.0-19.0)$ & $<0.001^{\ddagger}$ \\
\hline Presence of target mismatch $(\%)$ & $6(42.9)$ & $31(86.1)$ & $18(78.3)$ & $0.054^{+}$ \\
\hline Recanalization therapy, n (\%) & & & & 0.034 \\
\hline None & $4(28.6)$ & $1(2.8)$ & $4(17.4)$ & \\
\hline Intravenous & - & $4(11.1)$ & - & \\
\hline Endovascular & $2(14.3)$ & $13(36.1)$ & $4(17.4)$ & \\
\hline Combined & $8(57.1)$ & $18(50.0)$ & $15(65.2)$ & \\
\hline Onset to MR perfusion (min), median (IOR) & $192.5(117.5-286.8)$ & $149.5(117.3-199)$ & $193(138-247)$ & 0.247 \\
\hline Onset to groin puncture (min), median (IQR) & $194.5(157.5-232.5)$ & $220(170-290)$ & $250(220-320)$ & $0.048^{\S}$ \\
\hline Thrombolysis in cerebral infarction (TICI) grade, $n(\%)$ & & & & 0.968 \\
\hline $\mathrm{TICl} 0$ & $5(35.7)$ & $15(42.9)$ & $8(34.8)$ & \\
\hline $\mathrm{TICl} 1$ & $3(21.4)$ & $6(17.1)$ & $3(13.0)$ & \\
\hline $\mathrm{TICl} 2 \mathrm{a}$ & $1(7.1)$ & $2(5.7)$ & $2(8.7)$ & \\
\hline $\mathrm{TICl} 2 \mathrm{~b}$ & $3(21.4)$ & $6(17.1)$ & $7(30.4)$ & \\
\hline $\mathrm{TICl} 3$ & $2(14.3)$ & $6(17.1)$ & $3(13.0)$ & \\
\hline Infarct growth (mL), median (IOR) & $52.0(11.5-130.4)$ & $11.0(4.6-61.6)$ & $4.5(0.1-37.5)$ & $0.024^{\S}$ \\
\hline
\end{tabular}

MR, magnetic resonance; SD, standard deviation; NIHSS, NIH stroke scale.

${ }^{*}$ No significant difference after correction for multivariable comparisons; ${ }^{\dagger} P$ for trend; ${ }^{*}$ Statistically different between all subgroups, corrected for multivari-

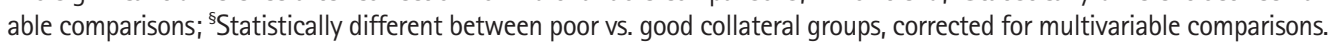


els were then applied to analyze the independent contribution of factors with univariate $P$ values of $<0.1$. A $P$ value of $<0.05$ was considered statistically significant.

\section{Results}

Of the 79 patients who met the inclusion criteria during the study period, 73 (92.4\%) patients were finally enrolled in this study. Six patients were excluded due to technically suboptimal imaging data: (a) the inability to generate a collateral flow map due to poor contrast bolus $(n=2)$, and (b) failure of MRI postprocessing for Tmax due to excessive patient motion or the absence of an identifiable, technically adequate arterial input function $(n=4)$. Baseline characteristics are presented in the Supplementary Table 1.

Of the 73 patients enrolled, 55 patients (75\%) showed a target mismatch pattern. The MRP-based collateral grading was poor in $14(19.2 \%)$ patients, intermediate in $36(49.3 \%)$, and good in $23(31.5 \%)$. The weight kappa $(\mathrm{kw})$ values for intra-observer agreement of the MRP-based collateral grade were 0.842 (95\% confidence interval $(\mathrm{Cl}), 0.733-0.950)$ and $0.851(95 \% \mathrm{Cl}$, 0.754-0.948) for the two reviewers respectively, while the $\mathrm{kw}$ value for inter-observer agreement was $0.817(95 \% \mathrm{Cl}$, 0.7040.931). Patient characteristics and the treatment response depending on the degree of collateral circulation are illustrated in Table 1. General characteristics were not different among the groups; except, current smoking was more prevalent and cardioembolism was less prevalent in patients with good collaterals. However, the baseline severity, as assessed by NIHSS score and DWI lesion volume, differed depending on the pretreatment collaterals. A trend was found between the collateral groups for the presence of a target-mismatch pattern ( $P$ for trend $=0.054$ ).

Although there was no significant difference in $\mathrm{TICl}$ grade among the collateral groups $(P=0.968)$ and the time interval between onset-to-treatment was shorter in the poor collateral group $(P=0.048)$, patients with poor collaterals definitely had larger infarct growth (median, interquartile range [IQR]; 52.0 [11.5-130.4] mL), compared with those with intermediate collaterals (11.0 [4.6-61.6] mL), or good collaterals $(4.5[0.1-37.5] \mathrm{mL})$ $(P=0.024)$. Post-hoc analysis revealed a significant difference in the volume of infarct growth between the poor and good collateral groups $(P=0.018)$.

Multivariable regression analysis was performed to evaluate the independent predictors for infarct growth further (Table 2). The attainment of recanalization represented by the $\mathrm{TICl}$ grade $(B=-16.7$, standard error mean $(S E)=3.65, P<0.001)$ and MRPbased collateral grading $(B=-15.8, S E=6.20, P=0.013)$ were independently associated with infarct growth, adjusting for other variables. Controlling for other factors, including age, initial severity of stroke (NIHSS score and DWI volume), glucose level, MR to groin puncture time, and the presence of target mismatch, did not significantly change the effect of the pretreatment collateral grade. When the MRP-based collateral grading was not entered into the same model, the presence of a target mismatch was not independently associated with infarct growth (data not shown).

Moreover, among patients who were homogenous in terms of the presence of a target mismatch and occurrence of recanalization, the degree of infarct growth was different depending on the MRP-based collateral grading. As shown in Figure 2, the degree of infarct growth in patients with a target mismatch pattern and successful recanalization differed depending on the collateral status. The volume of infarct growth was higher in patients with poor collaterals (median [IQR], 26 [16.6-33.9] mL) than those with intermediate $(7.8[-0.6-24.1] \mathrm{mL})$ or good $(1.8$ [-0.2-3.8] $\mathrm{mL}$ ) collaterals, although the difference is statistically insignificant due to the small sample size $(P=0.108)$.

A significantly lower volume of infarct growth was observed among patients in whom recanalization ( $\mathrm{TICl}$ grade 2-3) had been observed, regardless of collateral status and the presence of a target mismatch pattern (Figures 2 and 3B). Infarct growth, ex-

Table 2. Multivariate linear regression analysis for infarct growth

\begin{tabular}{|c|c|c|c|c|c|}
\hline & \multicolumn{2}{|c|}{ Univariate } & \multicolumn{2}{|c|}{ Multivariate } & \multirow{2}{*}{ Variance inflation factor } \\
\hline & $\mathrm{B}(S E)$ & $P$ value & $\mathrm{B}(S E)$ & $P$ value & \\
\hline Age & $0.43(0.451)$ & 0.345 & & & \\
\hline Initial NIHSS & $2.9(1.20)$ & 0.017 & $1.0(1.35)$ & 0.303 & 1.690 \\
\hline Initial DWI volume & $0.4(0.13)$ & 0.004 & $0(0)$ & 0.636 & 2.447 \\
\hline Target mismatch* & $-150.0(153.33)$ & 0.331 & $12.8(12.90)$ & 0.324 & 1.338 \\
\hline MR collateral status ${ }^{\dagger}$ & $-26.2(8.77)$ & 0.004 & $-15.8(6.20)$ & 0.013 & 1.501 \\
\hline Recanalization, $\mathrm{TICl}$ grade $^{\ddagger}$ & $-16.2(3.77)$ & $<0.001$ & $-16.7(3.65)$ & $<0.001$ & 1.068 \\
\hline MR to groin puncture (minute) & 3.1 (146.9) & 0.983 & & & \\
\hline Glucose level & $0.18(0.13)$ & 0.167 & & & \\
\hline
\end{tabular}

$\mathrm{SE}$, standard error mean; NIHSS, NIH stroke scale; DWI, diffusion-weighted imaging; MR, magnetic resonance; TICl, thrombolysis in cerebral infarction.

${ }^{*}$ Presence of target mismatch pattern; ${ }^{+}$Collateral grade: 1 vs. 2 vs. 3 vs. $4 ;{ }^{*} \mathrm{TICl}$ grade: 0 vs. 1 vs. $2 \mathrm{a}$ vs. $2 \mathrm{~b}$ vs. 3 . 

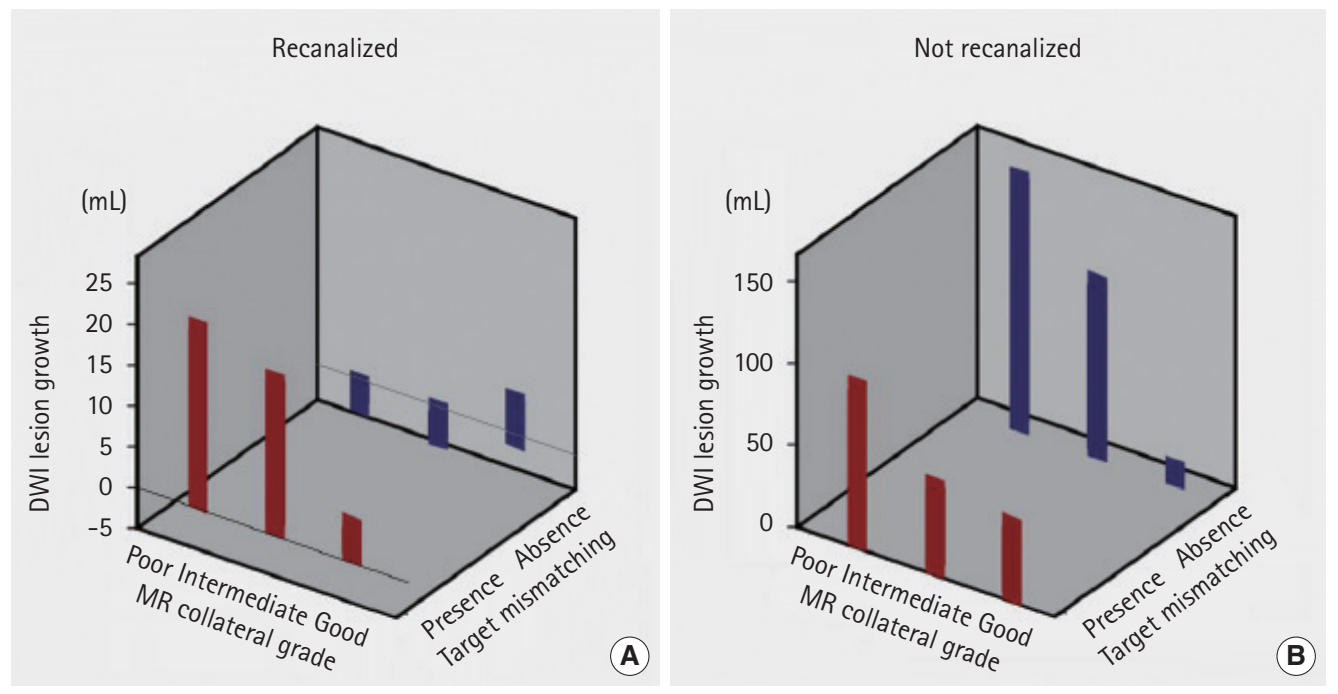

Figure 2. Degree of infarct growth depending on the collateral grade between recanalized and not recanalized.

pressed as median (IQR), in patients with recanalization vs. nonrecanalization was 10.4 (1.1-29.9) $\mathrm{mL}$ vs. 112.4 (69.7-148.5) $\mathrm{mL}$ in patients with poor collaterals $(P=0.001), 7.4(-1.4-23.5) \mathrm{mL}$ vs. 20.1 (5.3-99.4) $\mathrm{mL}$ in those with intermediate collaterals $(P=$ $0.041)$, and 0.5 (-0.5-4.1) $\mathrm{mL}$ vs. 37.5 (17.3-46.2) $\mathrm{mL}$ in those with good collaterals $(P<0.001)$. Similarly, infarct growth in patients with recanalization vs. non-recanalization was 4.6 (-0.123.5) $\mathrm{mL}$ vs. 25.6 (8.8-94.3) $\mathrm{mL}$ in patients with a target mismatch pattern $(P=0.001)$, and $-0.1(-6.4-10.5) \mathrm{mL}$ vs. 95.3 (36.2164.1) $\mathrm{mL}$ in those without a target mismatch pattern $(P=0.001)$.

Spearman's correlation analysis showed that degree of perfusion delay correlated significantly with MRP-based collateral grade throughout $T \max >2 \mathrm{~s}$ to $\operatorname{Tmax}>24 \mathrm{~s}$, but perfusion status at a later Tmax time points more closely correlated with collateral status (Figure 3A). Perfusion status at earlier Tmax time points was similar among patients with different collateral status. Figure 3B shows an example of a patient with good collaterals; although MRP showed extensive areas of Tmax delay up to 10 seconds, most of them were reperfused at a later Tmax time point.

\section{Discussion}

The main findings of the present study are as follows: (a) the attainment of early recanalization and the MRP-based collateral grading, but not the presence of a target mismatch pattern, were independently associated with infarct growth, (b) a target mismatch pattern on MRP was very common, and showed a poor correlation with pretreatment collaterals, and (c) collateral status was predicted by perfusion status at later Tmax time points but not by the extent of perfusion defects at earlier Tmax time points.

The hemodynamic effects of the collateral circulation are im- portant for maintaining perfusion to penumbral regions. ${ }^{21}$ In this study, there was a complex interplay between the extent of MRP-based collaterals, the presence of a target mismatch pattern, the attainment of recanalization, and the occurrence of infarct growth. Our data illustrate that collaterals and mismatch represent related, yet distinct, aspects of ischemic pathophysiology. There was a modest correlation between collateral grades and the presence of a target mismatch. Collaterals may influence the severity of ischemic injury, while the mismatch concept represents the extent of relatively mild hypoperfusion. Most currently used perfusion parameters such as time to peak, Tmax (deconvoluted time to peak), and mean time to transit have focused on the early part of the vascular phase (arterial and early capillary), whereas collateral evaluation (angiographic or MRPbased collateral maps) reflects the later phase (late capillary or venous). Our results showed that the perfusion status at later Tmax time points was more closely correlated with collateral status than that at earlier Tmax time points.

In the present study, the MRP-based collateral grading, but not the presence of a target mismatch pattern, were independently associated with infarct growth. Moreover, the degree of infarct growth differed depending on the collateral status, regardless of the presence or absence of a target mismatch pattern or successful recanalization. The results of the present study are in good agreement with our previous study showing that poor collaterals on conventional angiography are an important determinant of infarct growth, irrespective of the degree of recanalization. ${ }^{10}$ We used MRP-based collateral map, which can provide information about collateral circulation with good intra- and inter-observer agreement and avoid possible risk of thrombotic complications related to conventional angiography. ${ }^{16}$ 


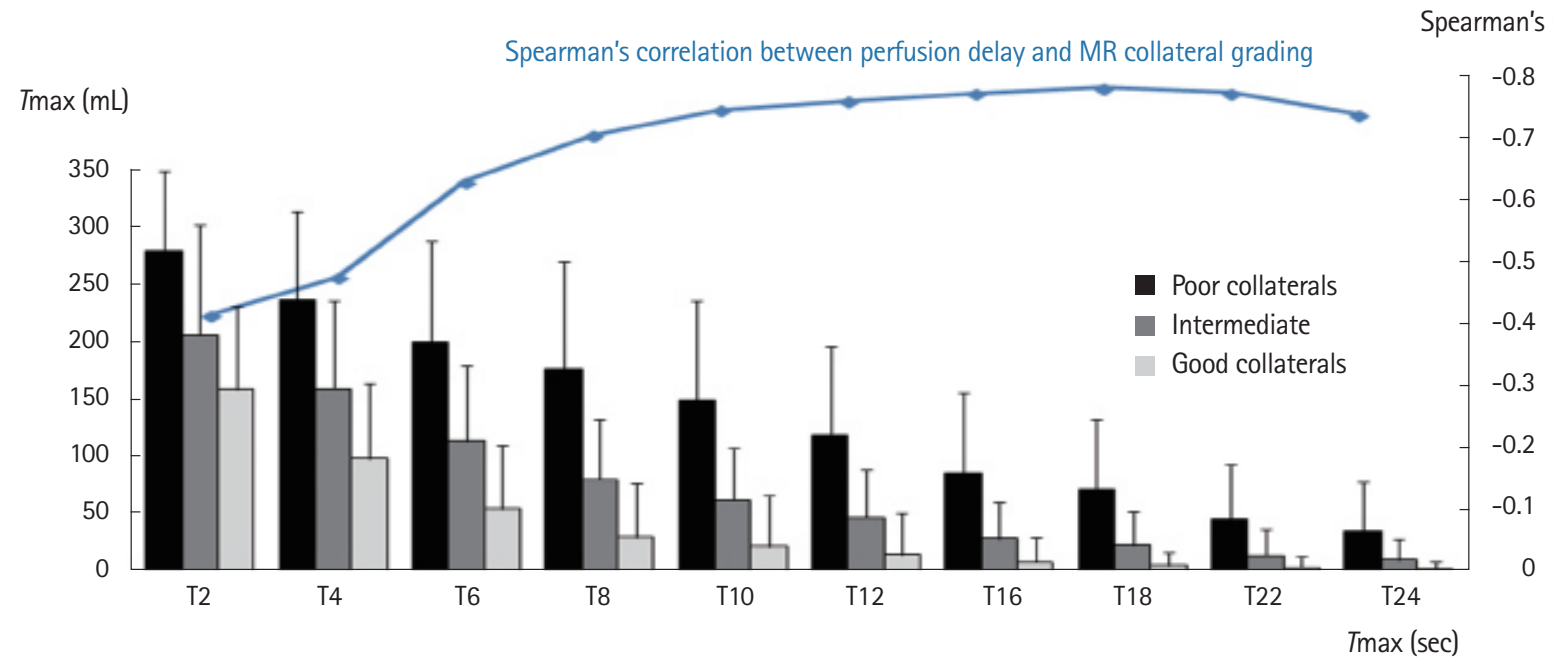

1. Tmax

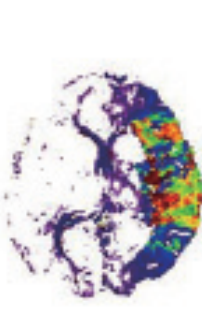

2. DWI

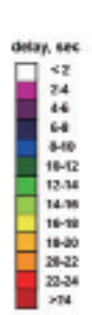

Initial

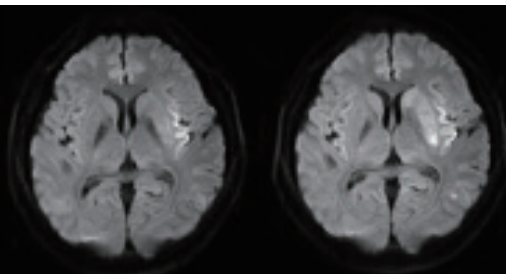

3. Collateral flow map Early phase Mid phase Late phase

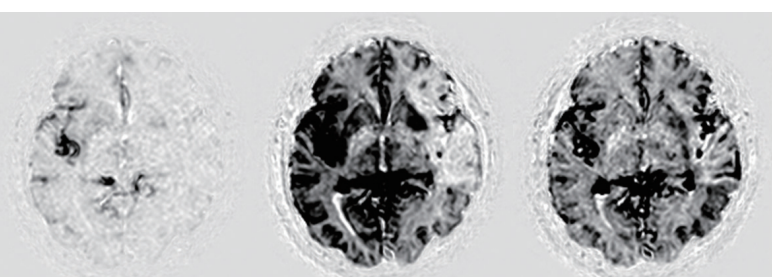

4. $T \max >2 s$ to 24

$T \max >2 \mathrm{~s}$ $>6 \mathrm{~s}$ $>8 \mathrm{~s}$ $>12 \mathrm{~s}$ $>16 s$ $>18 \mathrm{~s}$ $>22 \mathrm{~s}$ $>24 \mathrm{~s}$

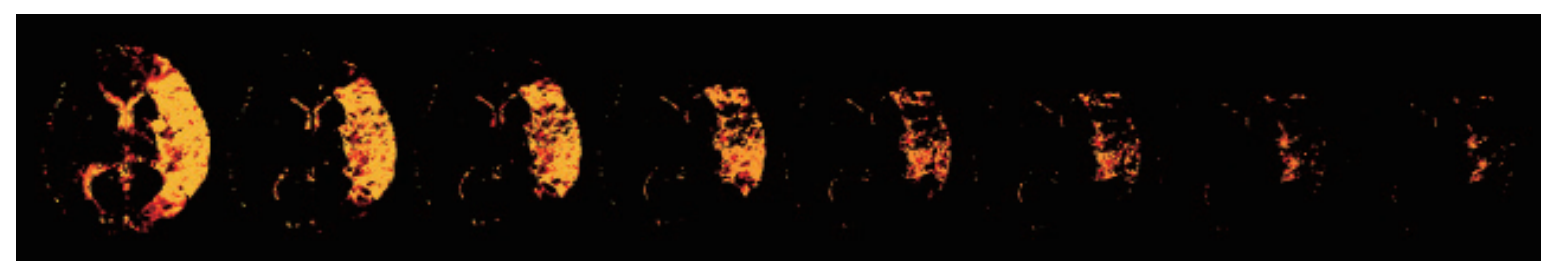

Figure 3. (A) Perfusion status depending on the collateral status and the spearman's correlation analysis of association of Tmax threshold and magnetic resonance perfusion (MRP)-based collateral grading. (B) Pretreatment (1) color-coded Tmax image, (2) diffusion-weighted imaging (DWI) images, and (3) collateral flow map findings in a patient with good collaterals (grade 3) on conventional angiography. Slow but almost complete blood filling was observed in (3) venous phase (right panel) of collateral flow map and (4) corresponding Tmax maps (Tmax 16-22 s).

However, it should be mentioned that MRI mismatch pattern is an MRI profile primarily used to identify patients that have more chances to benefit from treatment, rather than to predict clinical outcome. Moreover, although no significant association was observed between the collateral status and the target-mismatch pattern in the present study, collateral status is closely linked with perfusion-diffusion mismatch pattern. Results of others and our previous studies showed that better collateral flow at baseline was associated with larger perfusion-diffusion mismatch. ${ }^{22,23}$ Results of the present study indicate the importance of perfusion status at later Tmax time points (presence of slow blood filling via collateral flow) on infarct growth, rather than perfusion status at earlier Tmax time points. Our recent study of probabilistic approach with a Tmax-derived prediction model showed that Tmax severity-weighted model predicts poor collaterals with high predictive power. ${ }^{24}$ Therefore, image of collateral flow could provide additional information to perfusiondiffusion mismatch concept in the estimation of risk-benefits of revascularization therapy for acute ischemic stroke. Our results indicate that attainment of early recanalization is an important determinant of tissue fate, regardless of the presence of penumbral regions and the collateral grading. Therefore, in using MRI for acute stroke patients, it would be worthwhile to identify patients who are likely to be harmed by successful recanalization, rather than identifying those who are likely to show a favorable response to recanalization therapy. Besides a large DWI lesion 
volume, poor collaterals and the related severe hypoperfusion are reported to be associated with adverse effects (i.e., hemorrhagic transformation). ${ }^{12,19,25}$

This study has limitations. First, the results of this study should be interpreted with caution because of the limited sample size, and different treatment modalities. This was an observational study at a single medical center. Further prospective studies are warranted to fully validate the usefulness of MRP-based collateral evaluations. Second, our post-processing technique was different to that used in the DEFUSE-2 trial (RAPID software). Third, further studies using other imaging modalities to assess collateral status are needed to confirm our results. ${ }^{26}$ Lastly, some of the more substantial lesion growth in patients with poor collaterals may be related to the edema in patients with larger DWI lesions.

In conclusion, our results indicate that aside from the current diffusion-perfusion mismatch concept, the status of collateral flow generated from MRP source data may be a robust predictor of outcome in acute MCA stroke with large artery occlusion. Given that we used routine MRP source images in this study, the application of our method in a rapid prospective fashion is likely to be feasible in an acute clinical setting. A prospective study is ongoing to evaluate the impact of collateral flow on stroke outcome using this approach (clinical trial identifier NCT02668627 at clinicaltrial@gov). Although continuous efforts should be made to determine the optimal definition of penumbra, further studies are also needed to determine the optimal MRP parameters and thresholds for the measurement of collateral status.

\section{References}

1. Kidwell CS, Jahan R, Gornbein J, Alger JR, Nenov V, Ajani Z, et al. A trial of imaging selection and endovascular treatment for ischemic stroke. N Engl J Med 2013;368:914-923.

2. Hacke W, Furlan AJ, Al-Rawi Y, Davalos A, Fiebach JB, Gruber $F_{1}$ et al. Intravenous desmoteplase in patients with acute ischaemic stroke selected by MRI perfusion-diffusion weighted imaging or perfusion CT (DIAS-2): a prospective, randomised, double-blind, placebo-controlled study. Lancet Neurol 2009;8: 141-150.

3. Campbell BC, Mitchell PJ, Kleinig TJ, Dewey HM, Churilov L, Yassi N, et al. Endovascular therapy for ischemic stroke with perfusion-imaging selection. N Engl J Med 2015;372:10091018.

4. Albers GW, Thijs VN, Wechsler L, Kemp S, Schlaug G, Skalabrin $E$, et al. Magnetic resonance imaging profiles predict clinical response to early reperfusion: the diffusion and perfusion imaging evaluation for understanding stroke evolution (DEFUSE) study. Ann Neurol 2006;60:508-517.

5. Kakuda W, Lansberg MG, Thijs VN, Kemp SM, Bammer R, Wechsler LR, et al. Optimal definition for PWI/DWI mismatch in acute ischemic stroke patients. J Cereb Blood Flow Metab 2008;28:887-891.

6. Kidwell CS, Alger JR, Saver JL. Beyond mismatch: evolving paradigms in imaging the ischemic penumbra with multimodal magnetic resonance imaging. Stroke 2003;34:2729-2735.

7. Lansberg MG, Straka M, Kemp S, Mlynash M, Wechsler LR, Jovin $T G$, et al. MRI profile and response to endovascular reperfusion after stroke (DEFUSE 2): a prospective cohort study. Lancet Neurol 2012;11:860-867.

8. Nagakane Y, Christensen S, Ogata T, Churilov L, Ma H, Parsons $M W$, et al. Moving beyond a single perfusion threshold to define penumbra: a novel probabilistic mismatch definition. Stroke 2012;43:1548-1555.

9. Toth $\mathrm{G}$, Albers GW. Use of MRI to estimate the therapeutic window in acute stroke: is perfusion-weighted imaging/diffusion-weighted imaging mismatch an EPITHET for salvageable ischemic brain tissue? Stroke 2009;40:333-335.

10. Bang OY, Saver JL, Buck BH, Alger JR, Starkman S, Ovbiagele B, et al. Impact of collateral flow on tissue fate in acute ischaemic stroke. J Neurol Neurosurg Psychiatry 2008;79:625-629.

11. Bang OY, Saver JL, Kim SJ, Kim GM, Chung CS, Ovbiagele B, et al. Collateral flow predicts response to endovascular therapy for acute ischemic stroke. Stroke 2011;42:693-699.

12. Bang OY, Saver JL, Kim SJ, Kim GM, Chung CS, Ovbiagele B, et al. Collateral flow averts hemorrhagic transformation after endovascular therapy for acute ischemic stroke. Stroke 2011; 42:2235-2239.

13. Kucinski T, Koch $C$, Eckert B, Becker V, Krömer $H$, Heesen $C$, et al. Collateral circulation is an independent radiological predictor of outcome after thrombolysis in acute ischaemic stroke. Neuroradiology 2003;45:11-18.

14. Ribo M, Flores A, Rubiera M, Pagola J, Sargento-Freitas J, Rodriguez-Luna $D$, et al. Extending the time window for endovascular procedures according to collateral pial circulation. Stroke 2011;42:3465-3469.

15. Galimanis A, Jung S, Mono ML, Fischer U, Findling O, Weck A, et al. Endovascular therapy of 623 patients with anterior circulation stroke. Stroke 2012;43:1052-1057.

16. Kim SJ, Son JP, Ryoo S, Lee MJ, Cha J, Kim KH, et al. A novel magnetic resonance imaging approach to collateral flow imaging in ischemic stroke. Ann Neurol 2014;76:356-369.

17. Adams HP Jr, Bendixen BH, Kappelle ப, Biller J, Love BB, Gordon $D L$, et al. Classification of subtype of acute ischemic stroke. Definitions for use in a multicenter clinical trial. TOAST. Trial of Org 10172 in Acute Stroke Treatment. Stroke 1993;24: 
35-41.

18. Ostergaard L, Weisskoff RM, Chesler DA, Gyldensted C, Rosen $B R$. High resolution measurement of cerebral blood flow using intravascular tracer bolus passages. Part I: mathematical approach and statistical analysis. Magn Reson Med 1996;36:715725.

19. Kim JH, Bang OY, Liebeskind DS, Ovbiagele B, Kim GM, Chung CS, et al. Impact of baseline tissue status (diffusion-weighted imaging lesion) versus perfusion status (severity of hypoperfusion) on hemorrhagic transformation. Stroke 2010;41:e135e142.

20. Higashida RT, Furlan AJ, Roberts $H$, Tomsick T, Connors B, Barr $J$, et al. Trial design and reporting standards for intra-arterial cerebral thrombolysis for acute ischemic stroke. Stroke 2003; 34:e109-e137.

21. Liebeskind DS. Collateral circulation. Stroke 2003;34:22792284.

22. Campbell BC, Christensen S, Tress BM, Churilov L, Desmond $\mathrm{PM}$, Parsons MW, et al. Failure of collateral blood flow is asso- ciated with infarct growth in ischemic stroke. J Cereb Blood Flow Metab 2013;33:1168-1172.

23. Bang O, Saver J, Alger J, Starkman S, Ovbiagele B, Liebeskind $D$, et al. Determinants of the distribution and severity of hypoperfusion in patients with ischemic stroke. Neurology 2008;71: 1804-1811.

24. Lee MJ, Son JP, Kim SJ, Ryoo S, Woo SY, Cha J, et al. Predicting collateral status with MR perfusion parameters: probabilistic approach with a Tmax-derived prediction model. Stroke 2015; 46:2800-2807.

25. Campbell BC, Christensen S, Butcher KS, Gordon I, Parsons MW, Desmond PM, et al. Regional very low cerebral blood volume predicts hemorrhagic transformation better than diffusion-weighted imaging volume and thresholded apparent diffusion coefficient in acute ischemic stroke. Stroke 2010;41:8288.

26. Bang OY, Goyal M, Liebeskind DS. Collateral circulation in ischemic stroke: assessment tools and therapeutic strategies. Stroke 2015;46:3302-3309. 
Supplementary Table 1. General characteristics

\begin{tabular}{|c|c|}
\hline Female gender, n (\%) & $26(35.6)$ \\
\hline Age, year; mean $(S D)$ & $66.7(14.5)$ \\
\hline \multicolumn{2}{|l|}{ Risk factor, n (\%) } \\
\hline Atrial fibrillation & $32(43.8)$ \\
\hline Hypertension & $37(50.7)$ \\
\hline Diabetes & $17(23.3)$ \\
\hline Hyperlipidemia & $19(26.0)$ \\
\hline Current smoking & $10(13.7)$ \\
\hline Coronary artery disease & $9(12.3)$ \\
\hline Prior stroke & $20(27.4)$ \\
\hline \multicolumn{2}{|l|}{ Stroke mechanism } \\
\hline Atherosclerotic & $24(32.9)$ \\
\hline Cardioembolic & $34(46.6)$ \\
\hline Other & $6(8.2)$ \\
\hline Undetermined & $9(12.3)$ \\
\hline Initial NIHSS score, mean (SD) & $14.2(5.3)$ \\
\hline \multicolumn{2}{|l|}{ Occlusion site, $\mathrm{n}(\%)$} \\
\hline M1 & $43(58.9)$ \\
\hline Distal internal carotid artery & $18(24.7)$ \\
\hline Distal internal carotid artery+M1 & $12(16.4)$ \\
\hline \multicolumn{2}{|l|}{ Recanalization therapy, n (\%) } \\
\hline None & $9(12.3)$ \\
\hline Intravenous & $4(5.5)$ \\
\hline Endovascular & $19(26.0)$ \\
\hline Combined & $41(56.2)$ \\
\hline \multicolumn{2}{|l|}{ Recanalization, n (\%) } \\
\hline Thrombolysis in cerebral infarction ( $\mathrm{TICl}) 0$ & $28(38.9)$ \\
\hline $\mathrm{TICl} 1$ & $12(16.7)$ \\
\hline $\mathrm{TICl} 2 \mathrm{a}$ & $5(6.9)$ \\
\hline $\mathrm{TICl} 2 \mathrm{~b}$ & $16(22.2)$ \\
\hline $\mathrm{TICl} 3$ & 11 (15.3) \\
\hline
\end{tabular}

$\mathrm{SD}$, standard deviation. 


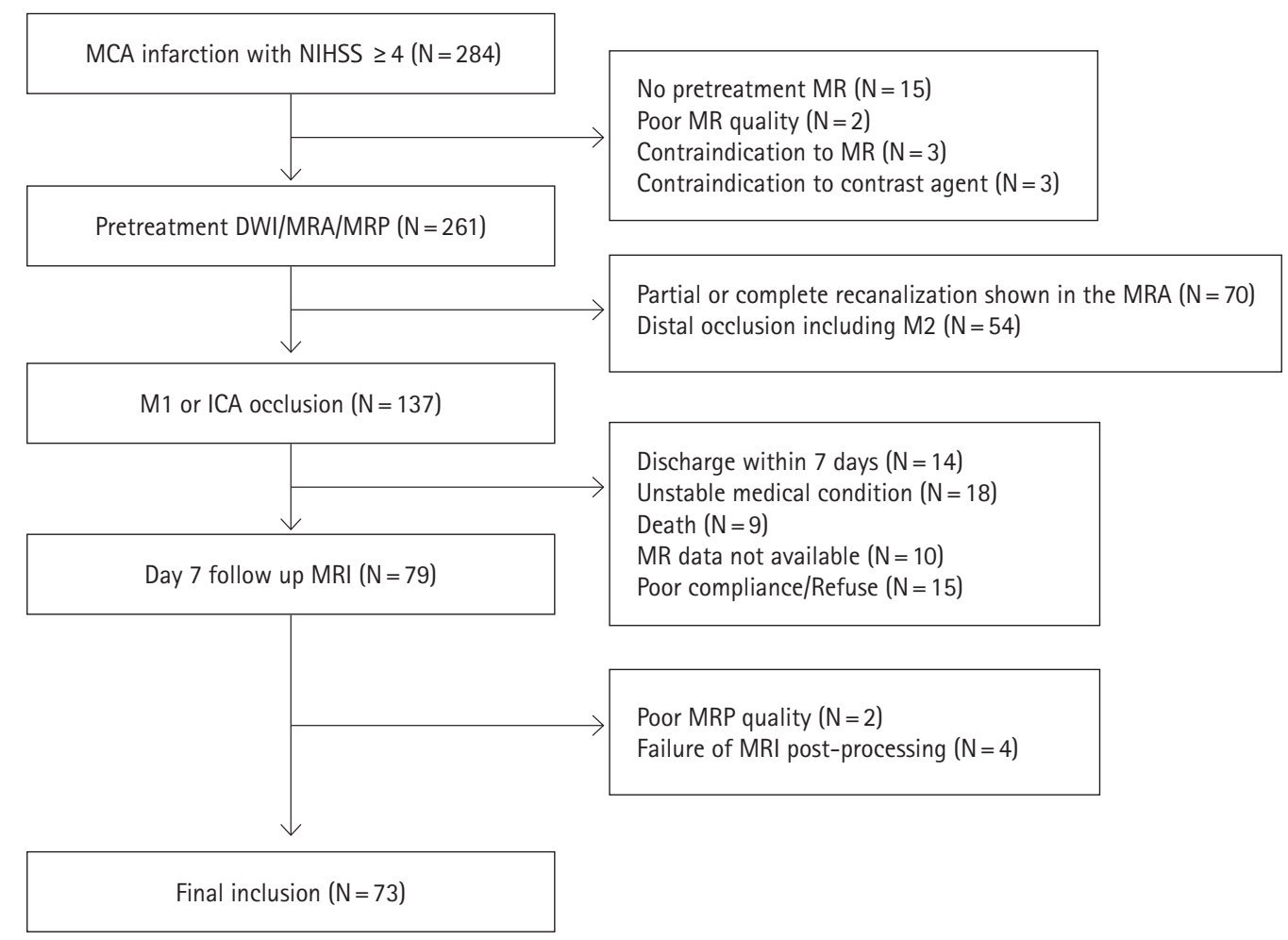

Supplementary Figure 1. Patient selection. 